\title{
ANÁLISE AMBIENTAL DE UMA ÁREA PARÁLICA NO DELTA DO RIO SÃO FRANCISCO, SERGIPE-BRASIL, COM BASE NA SINECOLOGIA DE FORAMINÍFEROS E TECAMEBAS (PROTISTA)
}

\author{
DÉCIOLUIS SEMENSATTO-JR \\ Programa de Pós-Graduação em Geociências e Meio Ambiente, IGCE, UNESP, Rio Claro, SP, Brasil. \\ semensattojr@terra.com.br \\ DIMAS DIAS-BRITO \\ LAMBdA, Depto. de Geologia Aplicada, IGCE, UNESP, Avenida 24A, 1515, Cx. Postal 178, \\ 13506-900, Rio Claro, SP, Brasil.dimasdb@rc.unesp.br
}

\begin{abstract}
RESUMO - Três conspícuos setores ambientais compõem a área parálica do extremo leste do Estado de Sergipe, Brasil, como evidenciado por estudos sinecológicos envolvendo foraminíferos e tecamebas. Nesta área, inserida no delta do rio São Francisco, três microbiofácies são reconhecidas: Quinqueloculina spp., Ammonia tepida/Elphidium spp. e Miliammina fusca/Arenoparrella mexicana. Tais unidades são fortemente controladas pela salinidade e correspondem, respectivamente, a sub-ambientes euhalinos $(33,5$ - 36,5\%o), hipohalinos-mixohalinos $(20,5$ - 28\%o) e hipohalinos-salobros $(0,5-9,5 \%$ ). Estes sub-ambientes integram um sistema formado por um canal lagunar, pequenos rios e canais de maré, que entrecortam manguezais. A diversidade e a eqüitatividade crescem em direção à laguna. Ambientes dominados por textulariinos e tecamebas são caracterizados por baixa diversidade, baixa eqüitatividade e alto grau de confinamento. Ambientes dominados por rotaliinos e milioliinos apresentam maior diversidade e eqüitatividade, além de serem mais abertos à influência marinha. Tais dados refletem o comportamento hidroquímico e hidrodinâmico da área e podem ser utilizados em estudos de diagnóstico ambiental e vulnerabilidade de ecossistemas. A zonação biofaciológica encontrada nesta área pouco impactada pode ser confrontada a áreas parálicas similares, inclusive poluídas, podendo também ser utilizada em estudos paleoambientais.
\end{abstract}

Palavras-chave: foraminíferos, tecamebas, manguezais, ambiente parálico, delta do rio São Francisco, Brasil.

ABSTRACT - ENVIRONMENTAL ANALYSIS OF A PARALIC AREA IN THE SÃO FRANCISCO RIVER DELTA, SERGIPE (BRAZIL), BASED ON FORAMINIFERA AND THECAMOEBIANS SYNECOLOGY. Three conspicuous environmental sectors compose the extreme eastern paralic area of the Sergipe State, Brazil, as disclosed by synecological study involving foraminifera and thecamoebians. In this area, a part of the São Francisco river delta, three main microbiofacies are distinguished: Quinqueloculina spp., Ammonia tepidalElphidium spp. and Miliammina fuscalArenoparrella mexicana. These biofacies are strongly controlled by salinity, and correspond, respectively, to euhaline $(33.5-36.5 \%$ ), hypohalinemixohaline $(20.5-28 \%$ ) and hypohaline-brackish $(0.5-9.5 \%$ ) sub-environments of a system that includes a lagoon channel and mangrove swamps intersected by small rivers and tidal creeks. Diversity and equitability values increase towards the lagoon. Environments dominated by textulariine and thecamoebians are characterized by low diversity, low equitability and high confinement degree. Milieux dominated by rotaliines and miliolines have higher diversity and equitability, and are more opened to marine influence. These data are reflecting the hydrochemical and hydrodynamics of the area and can be used in studies leading with environmental diagnosis and vulnerability of the ecosystem. The biofaciological zonation found in this relatively low impacted area can be applied in comparative studies involving similar paralic areas, including those that are polluted. It can be also used for paleoenvironmental purposes.

Key words: foraminifera, thecamoebians, mangrove swamps, paralic enviroment, São Francisco river delta, Brazil. 


\section{INTRODUÇÃO}

Nos últimos anos, as associações de foraminíferos parálicos vêm sendo cada vez mais estudadas em todo o mundo, tanto com o objetivo de se conhecer a ecologia destes protistas, quanto para auxiliar na compreensão do funcionamento natural dos ecossistemas e/ou detectar alterações neles induzidas pelas ações antrópicas (e.g., Scott \& Medioli, 1980; Sharifi et al., 1991; Alve, 1991, 1995; Brönnimann et al., 1992; Hayward \& Hollis, 1994; Schafer et al., 1995; Wetmore, 1995; Alve \& Murray, 1995, 1999; Donnici et al., 1997). Especificamente em relação ao Brasil, a maioria dos trabalhos tem se concentrado no estudo da porção sudeste e sul do país (e.g., Closs, 1962; Closs \& Madeira, 1966, 1967; Zaninetti et al., 1977; Brönnimann et al., 1981a,b; Brönnimann \& DiasBrito, 1982; Dias-Brito et al., 1988; Eichler et al., 1995; Eichler \& Bonetti, 1995; Bonetti, 1995; Debenay et al., 1998; Stevenson et al., 1998; Oliveira, 1999; Oliveira \& Dias-Brito, 1999;
Eichler, 2001; Debenay et al., 2001 a,b; Geslin et al., 2002; Duleba \& Debenay, 2003; Leipnitz et al., 2003). Em relação ao nordeste brasileiro destacam-se alguns trabalhos, como os de Zaninetti et al. (1979) e Zucon \& Loyola e Silva (1992), entre outros. Na maior parte destes estudos abordam-se aspectos relacionados aos padrões de distribuição e de resposta das espécies frente às flutuações das variáveis físicas e químicas.

O potencial de indicador ambiental e paleoambiental das tecamebas passou a ser mais estudado a partir da década de 1980, aparecendo hoje como ferramenta promissora para as análises de ecossistemas/paleoecossistemas de água doce e das faixas mais internas do sistema parálico (e.g., McCarthy et al., 1995; Laidler \& Scott, 1996; Oliveira \& Dias-Brito, 1999; Patterson \& Kumar, 2000a,b; Scott et al., 2001; Patterson et al., 2002).

No alto curso de estuários, estudos que analisam conjuntamente a distribuição das tecamebas e de foraminíferos au-
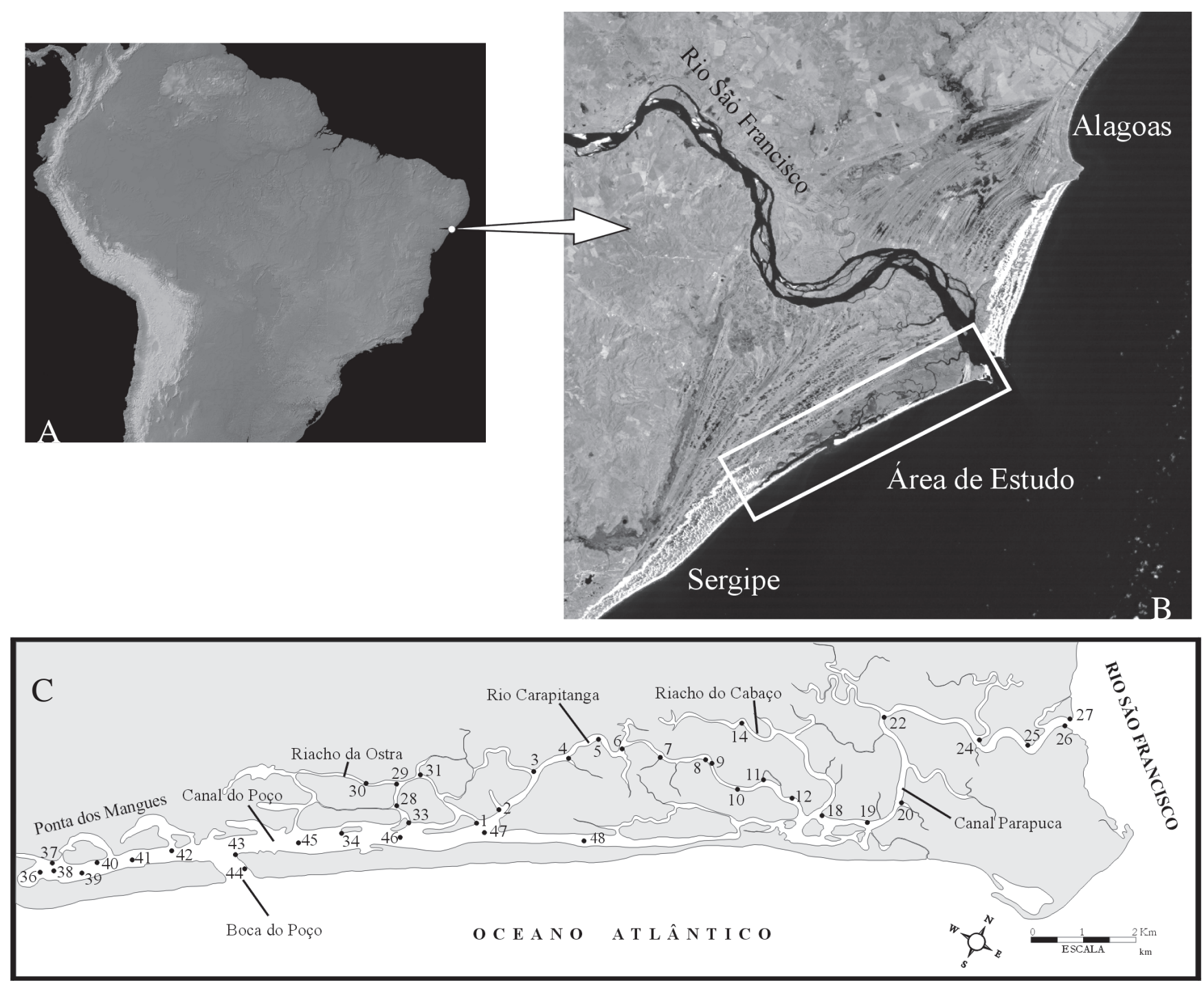

Figura 1. Área de estudo do projeto. A. Localização da área na América do Sul. B. Indicação do setor estudado no delta. C. Detalhamento do setor estudado com indicação das amostras.

Figure 1. Study area with sampling locations. A. Area location in South America. B. Study area in the delta. C. Study area in detail with sample locations. 
xiliam na compreensão do funcionamento do ecossistema, uma vez que as informações fornecidas por estes dois grupos são complementares entre si.

\section{Área de estudo}

A área de estudo, situada entre Ponta dos Mangues e a foz do Rio São Francisco, está submetida a um regime de micro-marés e está inserida no sistema deltaico do rio São Francisco, no extremo leste do Estado de Sergipe (Figura 1). Com aproximadamente $48,5 \mathrm{~km}^{2}$, tal área reúne um canal lagunar (canal do Poço) e pântanos cobertos por vegetação de mangue, cortados por uma rede de canais fluviais e de maré. Segundo a classificação de Boltovskoy et al. (1980), apresentada na Tabela 1, as águas variam de hipohalinassalobras a euhalinas (Figura 2). O inlet natural (Boca do Poço), que liga o mar à laguna, próximo às imediações de Ponta dos Mangues, exerce um importante papel na dinâmica do sistema, constituindo-se, hoje, no principal elemento desestabilizador de boa parte do ecossistema sob estudo. Em migração para oeste, ele vai propiciando o assoreamento do canal lagunar e de significativas áreas de manguezal (Dias-Brito, 1999). Esta migração dá-se em tal velocidade que pode ser constatada uma significativa diferença na posição do inlet em dois momentos distintos (compare a imagem de satélite, obtida há mais de uma década e o mapa em que o inlet foi posicionado em 1996 com o auxílio de GPS). O Canal Parapuca, que recebe água doce do São Francisco, é outro relevante elemento do sistema hidroquímico e hidrodinâmico da área.

Do ponto de vista da ação antrópica, a área estudada é relativamente bem preservada, embora já existam, sobretudo na região de Ponta dos Mangues, sinais de impactos importantes que são representados por retificações de canais e algum desmatamento. Santos (1997) afirma que os barramentos para geração de energia elétrica localizados à montante do São Francisco têm influenciado negativamente os regimes de vazão do rio, afetando os ecossistemas localizados na sua foz. O clima da região é caracterizado por altas temperaturas (média de $24^{\circ} \mathrm{C}$, com amplitudes menores que $4^{\circ} \mathrm{C}$ ) e pluviosidade concentrada no outono-inverno, principalmente no período de maio a julho. Os bosques de mangue da região, do tipo "ribeirinho", possuem alta produtividade e
Tabela 1. Classificação dos ambientes com base nos valores de salinidade (\%o), segundo Boltovskoy et al. (1980).

Table 1. Environmental classification based on salinity values (\%) according to Boltovskoy et al. (1980).

\begin{tabular}{cc}
\hline Salinidade & Tipo de Ambiente \\
\hline $40-75$ & Hiperhalino \\
$30-40$ & Euhalino \\
$18-30$ & Hipohalino-Mixohalino \\
$0,5-18$ & Hipohalino-Salobro \\
$<0,5$ & Doce \\
\hline
\end{tabular}

significância de potencial energético. De modo geral, Rhizophora mangle é a espécie dominante da franja dos bosques. Uma discussão sobre a distribuição das plantas da área, nas zonas de entre-maré e supra-maré, é apresentada em DiasBrito (1999).

\section{OBJETIVOS}

Este trabalho objetiva, em caráter preliminar, trazer subsídios para a compreensão do sistema de funcionamento desse setor do delta, sobretudo no que diz respeito ao seu regime hidroquímico e hidrodinâmico. Utiliza, para isso, uma análise sinecológica envolvendo foraminíferos e tecamebas. Busca também revelar as associações microfaunísticas parálicas que compõem esta faixa latitudinal da América do Sul que, como já há muito observou Boltovskoy (1965), ainda são relativamente pouco conhecidas. As contribuições de Rodrigues (1999) e de Dias-Brito \& Rodrigues (2000) representaram o início da investigação que está sob desenvolvimento. Pretende-se, aqui, oferecer informações auxiliares para estudos de diagnóstico e avaliação de vulnerabilidade ambiental da área e, ainda, gerar conhecimento a ser aplicado nas investigações paleoecológicas de bacias sedimentares costeiras.

\section{MATERIAL E MÉTODOS}

\section{Coleta e análise de laboratório}

As coletas foram realizadas em março de 1996 e as amostras referem-se aos $5 \mathrm{~cm}$ superficiais do sedimento, cada uma com um volume de $250 \mathrm{ml}$. No ato da coleta, anotou-

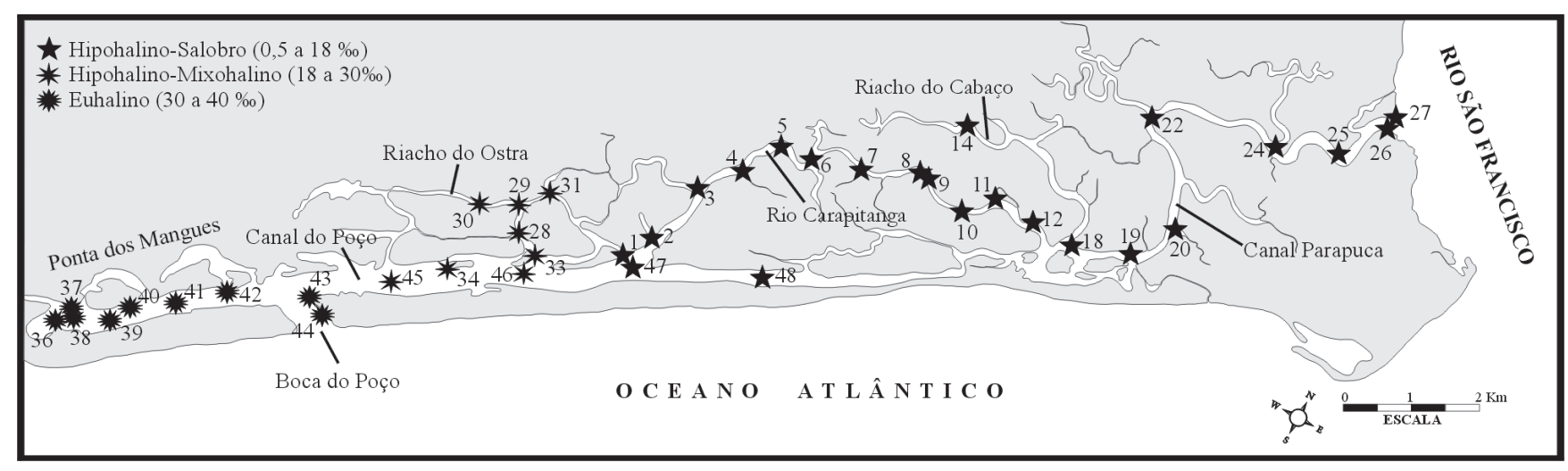

Figura 2. Valores de salinidade na área de estudo.

Figure 2. Salinity values at the study area. 
se a coordenada geográfica fornecida por GPS, além da tomada de uma alíquota de água para leitura da salinidade em laboratório, por meio de um eletrodo Corning PS18. As amostras 36 a 48 são relativas ao fundo da laguna, na zona de inframaré, e as demais foram coletadas nas margens dos canais estuarinos, junto ao manguezal, na zona de entremarés. Foram consideradas neste trabalho 40 amostras, sendo algumas previamente analisadas, embora com outro enfoque, por Rodrigues (1999) e Semensatto-Jr. (2001). As amostras foram estudadas do ponto de vista granulométrico e preparadas para triagem das tecas. Os $250 \mathrm{ml}$ de cada amostra foram peneirados com malhas conjugadas de 590 $\mu \mathrm{m}$ e $53 \mu \mathrm{m}$. A seguir, o material retido na última fração foi mergulhado em corante Rosa Bengala por duas horas. Após corado, o material foi seco em estufa a $50^{\circ} \mathrm{C}$ e flotado em tetracloreto de carbono $\left(\mathrm{CCl}_{4}\right)$, recolhendo-se o sobrenadante em papel-filtro. Por fim, as amostras foram armazenadas em frascos de vidro com solução de álcool 70\%. A triagem, ou separação das tecas dos restos orgânicos (sobretudo vegetais) e dos grãos minerais, foi feita a úmido com auxílio de pincel de pêlo marta $\mathrm{n}^{\circ} 00$, sob estereomicroscópio Wild Leica, com aumentos variando de 40 a 80x. Procurou-se obter 300 indivíduos em cada amostra, número estatisticamente significativo para análises embasadas nas espécies que representam, em abundância relativa, pelo menos 10\% da amostra total (Patterson \& Fishbein, 1989). Os indivíduos triados e identificados estão depositados na coleção do Laboratório de Análises Micropaleontológicas, Microbióticas e de Ambientes, LAMBdA, localizado na UNESP, em Rio Claro-SP. Embora tenha se utilizado corante na preparação das amostras a fim de reconhecer os indivíduos vivos no ato da coleta, optou-se por realizar a análise levando-se em conta a população total (indivíduos vivos e mortos), por melhor servir ao objetivo de distinguir zonas biofaciológicas.

Para identificação das espécies, consultou-se a coleção LAMBdA, além de trabalhos como os de Closs (1962), Boltovskoy et al. (1980), Ogden \& Hedley (1980), Todd \& Low (1981), Loeblich \& Tappan (1964, 1988); Scott et al. (1990), Brönnimann et al. (1992), Hayward \& Hollis (1994) e Oliveira (1999). Alguns espécimens foram separados para captura de imagens em MEV (Microscópio Eletrônico de Varredura), as quais foram obtidas no Centro de Pesquisas da Petrobras (CENPES/PETROBRAS), no Centro de Caracterização e Desenvolvimento de Materiais (UFSCar/UNESP) e no Instituto de Física da USP de São Carlos (SP).

\section{Tratamento estatístico}

Amostras com menos de 100 indivíduos não foram consideradas para os cálculos de diversidade e eqüitatividade. Para o cálculo do índice de confinamento, todas as amostras foram consideradas.

Dentre os vários índices de diversidade descritos na literatura (Magurran, 1988), optou-se pela utilização do índice de McIntosh modificado por Pielou (1969). Tal escolha apóiase no fato de que este índice dá maior peso às espécies dominantes, o que favorece o reconhecimento de comparti- mentos ambientais caracterizados por estas espécies (biofácies). Além disso, este índice é pouco influenciado pelo tamanho amostral e, comparado ao índice de Shannon, apresenta maior sensibilidade no agrupamento das amostras aqui estudadas (Semensatto-Jr., 2003). Segue-se a fórmula inicialmente proposta por McIntosh (1967):

$$
U=\sqrt{\sum n_{i}^{2}}
$$

Nesta fórmula, $n_{i}$ é a abundância da $i$-ésima espécie. A fórmula modificada por Pielou (1969) é a que se segue:

$$
D=\frac{N-U}{N-\sqrt{N}}
$$

Na equação anterior, $N$ é o somatório das abundâncias de todas as espécies na amostra, e $U$ é dado pela equação (1). Nos cálculos de diversidade aqui realizados foi utilizada a equação (2).

A eqüitatividade, i. e., a homogeneidade da distribuição proporcional das abundâncias das espécies em uma comunidade, é dada a seguir (Pielou, 1969):

$$
E=\frac{N-U}{N-\frac{N}{\sqrt{S}}}
$$

Na equação (3), $N$ é a abundância total da amostra, $U$ é dado pela equação (1) e $S$ é o número total de espécies da amostra.

As proporções entre as espécies foram utilizadas no cálculo do índice de confinamento (Debenay, 1990), que reflete o grau de influência marinha sobre o ambiente:

$$
I_{C}=\frac{\left(\frac{C}{B+C}-\frac{A}{A+B}\right)+1}{2}
$$

Nesta última fórmula, $A$ representa a soma das frequiências relativas das espécies típicas de ambientes marinhos costeiros (e. g., Brizalina striatula, Bulimina marginata); $B$ representa a soma das frequiências relativas das espécies de ambientes marinhos moderadamente confinados (e. g., Quinqueloculina seminulum, Ammonia tepida, Elphidium excavatum); e $C$ representa a soma das freqüências relativas das espécies típicas de ambientes com forte confinamento (e. g., Miliammina fusca, Arenoparrella mexicana, Haplophragmoides wilberti, Ammobaculites exiguus). Ambientes com valores de $I_{C}$ entre 0 e 0,4 são considerados como marinhos; entre 0,4 e 0,7 são pouco restritos à influência marinha; entre 0,7 e 0,9 são restritos à influência marinha; e entre 0,9 e 1 são confinados.

Para as amostras com mais de 100 indivíduos, os dados de salinidade, diversidade, eqüitatividade e confinamento foram empregados em uma análise de agrupamento, realizada no programa Statistica. Todos os dados foram transformados 
por centro-redução. O método das ligações médias usando o algoritmo UPGMA (unweighted pair group method using arithmetic averages) e a distância euclidiana foram utilizados para avaliar a similaridade entre as amostras.

\section{RESULTADOS E DISCUSSÃO}

Nas 40 amostras estudadas, representadas por sedimentos lamosos, lamo-arenosos, areno-lamosos ou argi- losos, foram encontradas 63 espécies de foraminíferos bentônicos, num total de 7.239 indivíduos. Apenas as amostras 43, 44 e 45 não apresentaram espécimen algum. Estão representadas as sub-ordens Textulariina (com 39 espécies), Rotaliina (18 espécies), Miliolina (4 espécies), Lagenina e Allogromiina (1 espécie cada). Quanto às tecamebas, foram encontradas 18 espécies, num total de 428 indivíduos, todas pertencentes à superfamília Arcellacea.

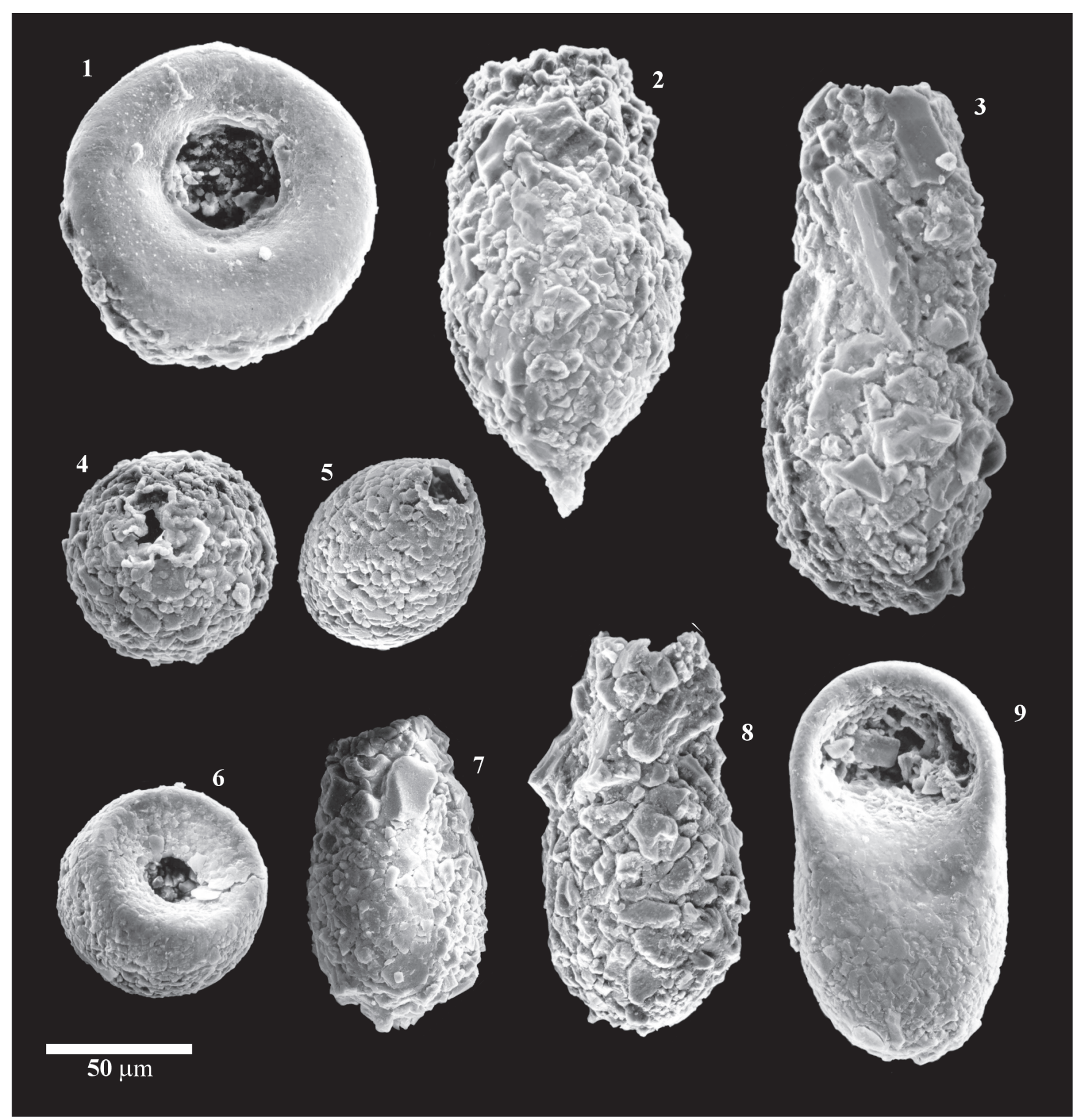

Figura 3. Algumas espécies de tecamebas encontradas no canal Parapuca. 1. Centropyxis aculeata; 2. Difflugia protaeiformis; 3. Lagenodifflugia vas; 4,5 . Cucurbitella tricuspis; 6. Cyclopyxis sp.; 7. Difflugia viscidula; 8. Difflugia oblonga; 9. Centropyxis constricta. Figure 3. Some of the thecamoebian species found in the Parapuca channel. 


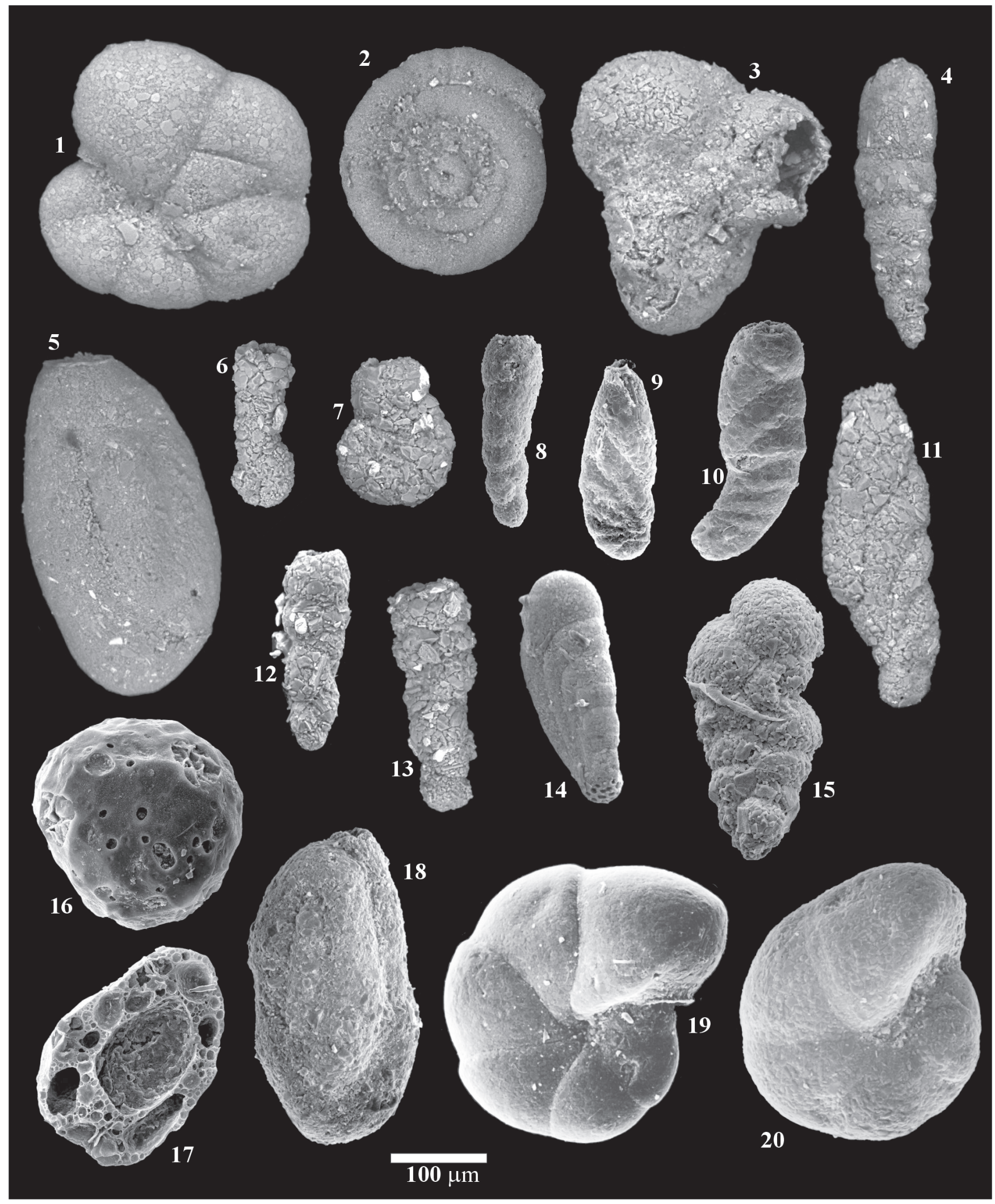

Figura 4. Algumas espécies de foraminíferos textulariinos e alogromiinos encontrados nos canais Parapuca e Carapitanga. 1. Haplophragmoides manilaensis; 2. Ammodiscus sp.; 3. Trochamminita irregularis; 4. Warrenita palustris; 5. Miliammina fusca; 6, 7. Ammobaculites exiguus; 8. Monotalea salsa; 9. Ammotium cassis; 10. Ammotium salsum; 11. Ammotium pseudocassis; $12,13$. Polysaccammina hyperhalina; 14. Ammoastuta inepta; 15. Textularia earlandi; 16, 17. Blysmasphaera brasiliensis; 18. Miliammina fusca; 19. Haplophragmoides wilberti; 20. Arenoparrella mexicana.

Figure 4. Some of the textulariine and alogromiine foraminifers found in the Parapuca and Carapitanga channels. 


\section{Os canais Parapuca e Carapitanga}

Os canais Parapuca e Carapitanga são ambientes similares em termos de estrutura da microfauna de foraminíferos. Todavia, o Parapuca se diferencia por conter várias espécies de tecamebas, algumas delas apresentadas na Figura 3. Num contexto geral, domina Miliammina fusca, sempre seguida por Arenoparrella mexicana. A exceção fica apenas para o ponto 27, onde domina Centropyxis aculeata. Bonetti (1995) e Oliveira (1999) relatam que em Cananéia, Itanhaém e outras áreas, esta é uma das espécies de tecamebas mais tolerantes

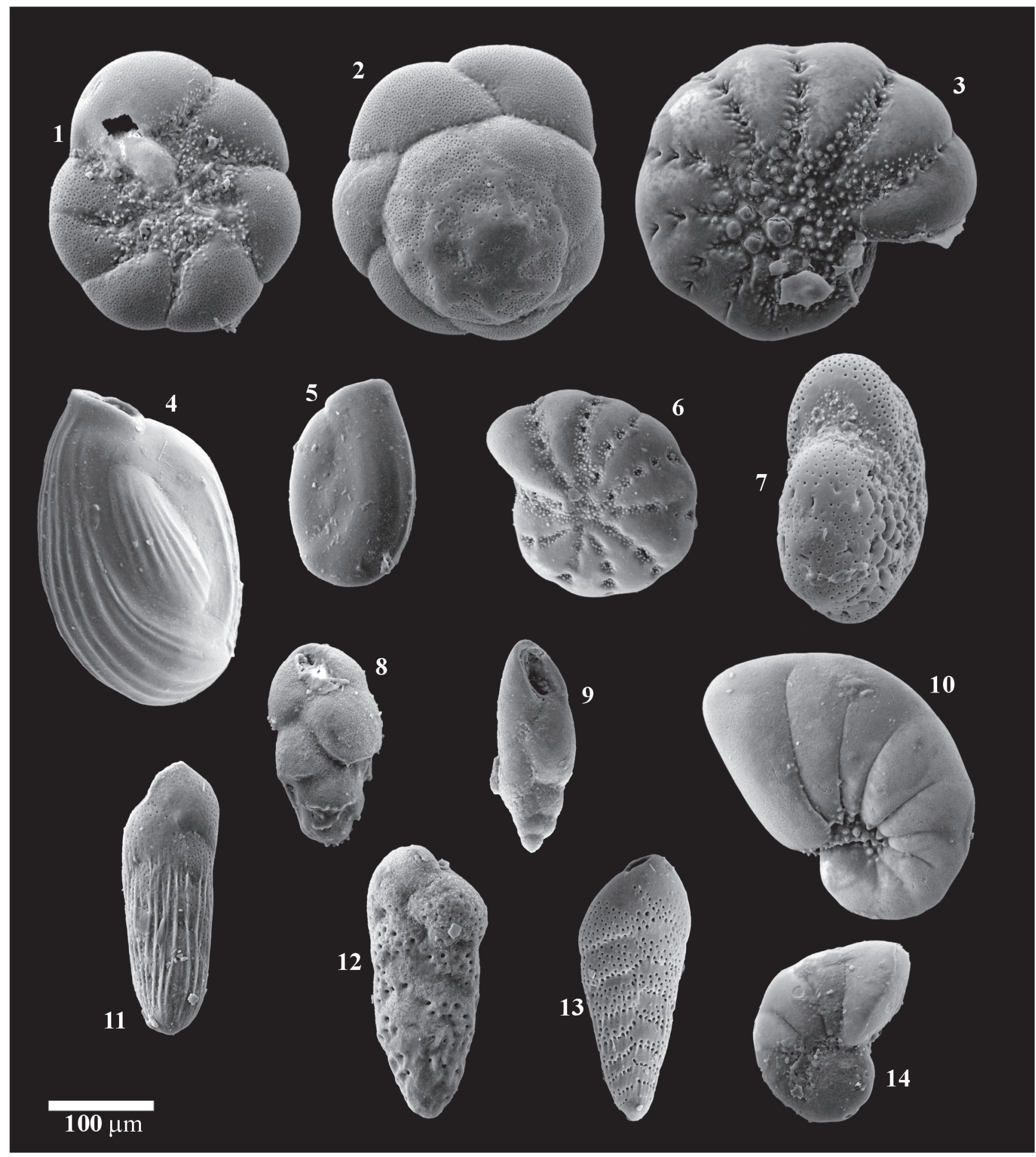

Figura 5. Algumas espécies de foraminíferos rotaliinos e miliolinos do Canal do Poço. 1, 2. Ammonia tepida; 3. Elphidium excavatum; 4. Quinqueloculina sp. 1; 5. Quinqueloculina sp. 2; 6. Elphidium sp. 1; 7. Elphidium sp. 2; 8. Bulimina marginata; 9 . Fursenkoina sp.; 10. Pseudononion atlanticum; 11. Brizalina striatula; 12. Bolivina sp. 1; 13. Bolivina sp. 2; 14. Pseudononion atlanticum.

Figure 5. Some of the rotaliine and milioline foraminifers found at the Canal do Poço. 
à salinidade. Além de M. fusca e A. mexicana, Ammotium cassis, Haplophragmoides wilberti e Trochammina inflata ocorrem em quase todas as amostras dos dois canais. Estas espécies são comumente relatadas por autores que executam pesquisas sinecológicas de foraminíferos em regiões estuarinas e, nesses canais, acham-se associadas a dezenas de táxons (Zaninetti et al., 1979; Brönnimann et al., 1981b; Hayward \& Hollis, 1994; Barbosa, 1995; Bonetti, 1995; Oliveira, 1999; Eichler, 2001; Duleba \& Debenay, 2003), alguns deles mostrados na Figura 4.

A diversidade e a eqüitatividade dos canais Parapuca e Carapitanga são relativamente baixas, sendo que apenas em pontos onde há a presença significativa das tecamebas, ou na transição Carapitanga/canal do Poço, os valores de tais variáveis são similares aos encontrados na laguna. Este fato ocorre porque estes pontos marcam a transição entre diferentes ambientes (como uma forma de "ecótono"). Assim, há mistura ou superposição das faunas típicas dos

Tabela 2. Variáveis utilizadas no trabalho. $D$ : índice de diversidade de Mclntosh modificado por Pielou (1969); E: equitatividade; S: riqueza de espécies; $n$ : abundância absoluta de indivíduos; $I_{C}$ : índice de confinamento; Salinidade (\%o); Argila/Silte (\%).

Table 2. Variables used in this work. $D$ : Mclntosh's diversity index; $E$ : equitability; $S$ : species richness; $n$ : absolute abundance of specimens; $I_{C}$ : confining index; Salinity $(\%)$; Mud (\%).

\begin{tabular}{|c|c|c|c|c|c|c|c|c|}
\hline Ambiente & Amostra & D & $E$ & $\mathbf{s}$ & $n$ & Ic & $\begin{array}{c}\text { Salini- } \\
\text { dade }\end{array}$ & $\begin{array}{c}\text { Argilal } \\
\text { Silte }\end{array}$ \\
\hline \multirow{12}{*}{ 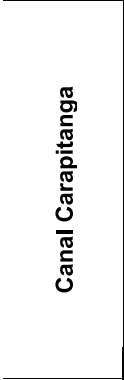 } & 1 & - & - & 14 & 46 & 0,76 & 13 & 54 \\
\hline & 2 & 0,6 & 0,7 & 22 & 160 & 0,98 & 9,5 & 96 \\
\hline & 3 & 0,3 & 0,4 & 15 & 213 & 0,99 & 7,5 & 58 \\
\hline & 4 & 0,4 & 0,5 & 19 & 302 & 0,99 & 4,5 & 71 \\
\hline & 5 & 0,5 & 0,6 & 15 & 203 & 1 & 4,5 & 94 \\
\hline & 6 & 0,1 & 0,2 & 10 & 309 & 1 & 4,5 & 84 \\
\hline & 7 & 0,1 & 0,1 & 6 & 302 & 1 & 2,7 & 93 \\
\hline & 8 & 0,0 & 0,0 & 4 & 95 & 1 & 1,4 & 98 \\
\hline & 9 & 0,3 & 0,5 & 9 & 292 & 1 & 0,96 & 89 \\
\hline & 10 & 0,4 & 0,5 & 11 & 99 & 0,99 & 1,36 & 88 \\
\hline & 11 & 0,1 & 0,1 & 5 & 142 & 1 & 1,53 & 93 \\
\hline & 12 & 0,2 & 0,3 & 6 & 169 & 1 & 1,65 & 67 \\
\hline $\begin{array}{c}\text { Riacho do } \\
\text { Cabaco }\end{array}$ & 14 & 0,3 & 0,4 & 13 & 204 & 1 & 1,22 & 95 \\
\hline \multirow{8}{*}{ 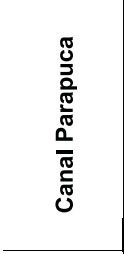 } & 18 & 0,1 & 0,2 & 11 & 309 & 1 & 1,8 & 84 \\
\hline & 19 & 0,4 & 0,5 & 20 & 400 & 1 & 3 & 80 \\
\hline & 20 & 0,3 & 0,4 & 21 & 203 & 1 & 2,6 & 84 \\
\hline & 22 & 0,3 & 0,4 & 14 & 299 & 1 & 2,6 & 25 \\
\hline & 24 & 0,1 & 0,1 & 12 & 300 & 1 & 2,8 & 94 \\
\hline & 25 & 0,1 & 0,2 & 15 & 299 & 1 & 1,1 & 61 \\
\hline & 26 & 0,5 & 0,6 & 20 & 289 & 1 & 1,7 & 66 \\
\hline & 27 & 0,6 & 0,7 & 23 & 209 & 1 & 0,5 & 26 \\
\hline \multirow{4}{*}{ 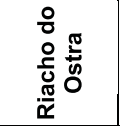 } & 28 & 0,6 & 0,8 & 15 & 245 & 0,82 & 26,5 & 58 \\
\hline & 29 & 0,7 & 0,8 & 18 & 219 & 0,92 & 24,5 & 71 \\
\hline & 30 & - & - & 12 & 42 & 0,94 & 27 & 82 \\
\hline & 31 & 0,4 & 0,6 & 15 & 216 & 0,97 & 23 & 89 \\
\hline \multirow{12}{*}{$\begin{array}{l}\text { 응 } \\
0 \\
0 \\
\frac{0}{0} \\
\overline{0} \\
\overline{0} \\
0\end{array}$} & 33 & 0,5 & 0,6 & 15 & 435 & 0,52 & 28 & 43 \\
\hline & 34 & 0,3 & 0,3 & 19 & 181 & 0,58 & 25,2 & 56 \\
\hline & 36 & 0,7 & 0,8 & 14 & 138 & 0,48 & 33,5 & 94 \\
\hline & 37 & - & - & 2 & 14 & 0,5 & 33,5 & 65 \\
\hline & 38 & - & - & 6 & 30 & 0,5 & 33,5 & 99 \\
\hline & 39 & - & - & 3 & 7 & 0,43 & 35 & 97 \\
\hline & 40 & 0,7 & 0,9 & 12 & 323 & 0,48 & 35,8 & 67 \\
\hline & 41 & 0,4 & 0,6 & 10 & 320 & 0,49 & 36,5 & 39 \\
\hline & 42 & 0,4 & 0,5 & 14 & 304 & 0,5 & 35 & 66 \\
\hline & 46 & 0,5 & 0,6 & 11 & 312 & 0,5 & 20,5 & 6 \\
\hline & 47 & & - & 8 & 21 & 1 & 17,5 & 25 \\
\hline & 48 & - & - & 6 & 16 & 1 & 13 & 14 \\
\hline
\end{tabular}

ambientes adjacentes, aumentado a riqueza de espécies. Como nessas transições os níveis de dominância são menores e a abundância proporcional das espécies é melhor distribuída, os valores de diversidade e eqüitatividade aumentam.

Quanto à influência marinha, os canais Parapuca e Carapitanga devem ser considerados como "confinados". Este dado reflete a contribuição expressiva das águas do rio São Francisco na manutenção dos níveis constatados de salinidade. Em outras regiões, o mesmo grau de confinamento só é encontrado a quilômetros do litoral. Na costa noroeste africana, nas áreas parálicas do Senegal, a isolinha de 0,9 mais próxima do litoral aparece $35 \mathrm{~km}$ à montante, como relata Debenay (1990). Em Cananéia, Bonetti (1995) encontrou tal valor a pouco mais de $20 \mathrm{~km}$ do litoral. A mesma autora cita o Estuário de Guaratuba, estudado por Barbosa (1995), em que a isolinha de 0,9 está a 7,5 km da costa. Já no rio São Francisco esta isolinha está a menos de $1 \mathrm{~km}$ do litoral. Assim, é possível reconhecer que, apesar da alteração da vazão do rio São Francisco provocada pelos barramentos à montante, como relata Santos (1997), a contribuição das águas vindas do rio é expressiva em sua margem direita. A mesma observação é feita por Dias-Brito \& Rodrigues (2000) com base na estrutura da flora presente às margens do Parapuca.

\section{O canal lagunar "canal do Poço" e o riacho do Ostra}

O canal do Poço é o setor que recebe a maior influência marinha de todo o sistema analisado. Tal fato é refletido por todas as variáveis estudadas nesse trabalho. Ali são registrados os mais elevados níveis de salinidade, enquanto a diversidade e a eqüitatividade microfaunística atingem seus mais altos valores. Nas imediações de Ponta dos Mangues tal influência é particularmente marcante, já que nos sedimentos dominados por Quinqueloculina spp. estão presentes espécies de foraminíferos de águas marinhas normais (e. g., Lagena sp., Bolivina spp., Bulimina marginata, Fursenkoina sp.). Este fato mostra que o inlet que liga a laguna ao mar é uma eficiente porta de entrada de águas euhalinas para aquele setor, não havendo, por outro lado, aporte significativo de água doce naquela área. Próximo à transição do canal lagunar para o Carapitanga, a influência marinha decresce e a dominância é exercida por Ammonia tepida e Elphidium spp., quase desaparecendo nas imediações dos pontos 47 e 48 , onde ocorrem apenas tecamebas (Arcella sp., Cyclopyxis constricta, Cyclopyxis sp., Difflugia sp., Lagunculina sp.) e foraminíferos textulariinos típicos de ambientes hipohalino-salobros (e. g., Arenoparrella mexicana, Miliammina fusca, Trochammina inflata, Ammobaculites exiguus). A Figura 5 mostra alguns dos principais táxons encontrados no canal do Poço. O riacho do Ostra representa um ambiente transicional, sob influência marinha um pouco mais acentuada.

\section{Caracterização das biofácies}

Os dados bióticos e abióticos constantes da Tabela 2 foram integrados numa análise de agrupamento para 


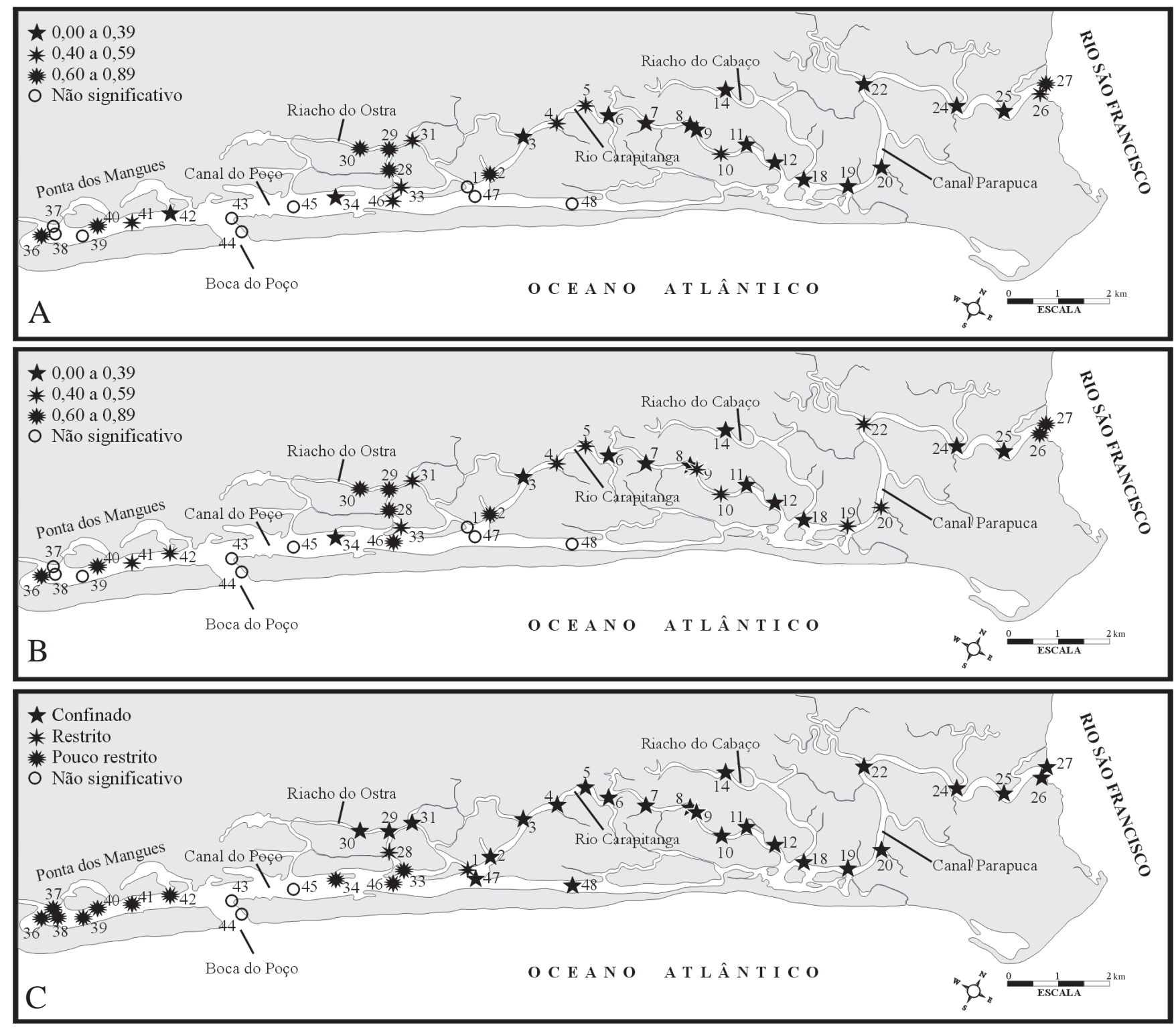

Figura 6. Distribuição das variáveis bióticas na área de estudo. A. Diversidade de Mclntosh modificado por Pielou; B. Eqüitatividade; C. Índice de confinamento.

Figure 6. Distribution of biotic variables at the study area. A. McIntosh's diversity modified by Pielou (1969); B. Equitability; C. Confining index.

reconhecimento das diferentes biofácies. A Figura 6 ilustra a distribuição das variáveis bióticas na área de estudo.

Observando-se o dendrograma na Figura 7, é possível reconhecer três domínios distintos no nível de corte de 2,4, os quais podem ser considerados como: biofácies Quinqueloculina spp., correspondente ao domínio lagunar euhalino, no qual se observa o agrupamento das amostras de Ponta dos Mangues (36 e 40); biofácies Ammonia tepidal Elphidium spp., que corresponde ao domínio mixohalino, localizado na região central e nordeste da laguna próximo ao Carapitanga, além do riacho da Ostra (amostras 28, 29, 30, 31, 33, 34 41, 42 e 46); e biofácies Miliammina fuscal Arenoparrella mexicana, representada pelo domínio confinado hipohalino-salobro, composto pelas amostras dos canais Carapitanga e Parapuca.
A análise isolada da biofácies $M$. fusca/A. mexicana revela dois subgrupos distintos. O primeiro, formado pelas amostras $6,7,8,11,18,24$ e 25 se diferencia das demais pelos baixos valores de diversidade. O segundo grupo é constituído pelas demais amostras dos canais. Os pontos 2 e 27 refletem um comportamento transicional, pois seu agrupamento é menos forte e estão mais distantes das amostras restantes. Como essas amostras estão localizadas nos extremos da biofácies, há aumento nos valores de diversidade causado pela "mistura" com a microfauna das biofácies adjacentes. Rodrigues (1999) discute que, se considerando apenas o canal Parapuca, as amostras 25 e 26 também podem ser enquadradas no aspecto transicional com a amostra 27, pois apresentam alguns táxons tolerantes a níveis de salinidades maiores que se distribuem apenas nesta área (e.g., Trochammina inflata, Trochammina 


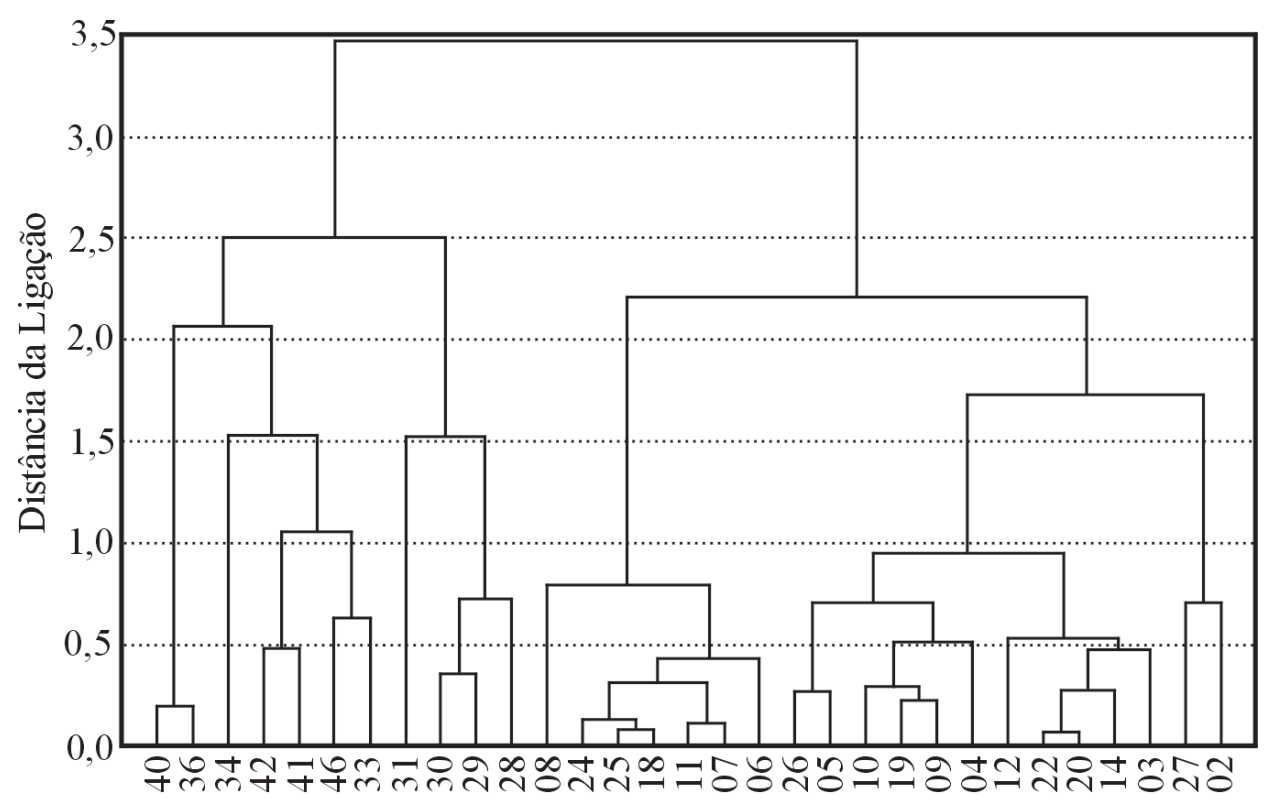

Figura 7. Dendrograma obtido a partir da análise de agrupamento das amostras.

Figure 7. Tree diagram obtained from cluster analysis.

cf. macrescens macrescens, Trochammina macrescens polystoma, Textularia earlandi, Tiphotrocha comprimata, Ammobaculites exiguus e Glomospira gordialis). Isto é efeito de uma cunha salina mesohalina a polihalina que deve atingir freqüentemente os sedimentos da zona de entre-maré desta área. Nos períodos de preamar, quando a cunha se dirige em direção à montante do rio São Francisco, as margens de sua massa d'água devem alcançar a desembocadura do Parapuca. Como a cunha percorre o fundo e não é capaz de inverter o sentido da vazão do rio, sua massa mais densa e mais pesada funciona como uma barreira subsuperficial ao sentido normal de vazão do rio, e deve provocar o "espalhamento" da água doce superficial em direção às margens (Figura 8). Assim, o canal Parapuca recebe em seu interior um aporte significativo de águas hipohalina-doces, embora sua desembocadura permaneça mais influenciada pela cunha salina. Esta dinâmica é responsável pela manutenção de condições favoráveis ao desenvolvimento de tecamebas, que alcançam nesse ambiente os maiores valores de abundância e riqueza de espécies.

Ao observar as similaridades dos domínios euhalinos e hipohalino-mixohalinos (biofácies Quinqueloculina spp. e Ammonia tepida/Elphidium spp., respectivamente), percebe-se que as distâncias entre as amostras são maiores, comparadas às do domínio confinado hipohalino-salobro (biofácies $M$. fusca/A. mexicana). Isto ocorre porque a laguna abriga maior riqueza de espécies, sendo que as comunidades se estruturam de forma mais complexa. Deste modo, há maior variabilidade na organização da microfauna, o que é refletido na menor similaridade entre as amostras.

Embora faça parte da biofácies Ammonia tepida/Elphidium spp., o riacho da Ostra (amostras 28, 29, 30 e 31) apresenta menor similaridade com as demais amostras desse domínio. Esta distância é determinada essencialmente pelo maior grau de confinamento deste ambiente, pois é composto essencialmente por textulariinos. Entretanto, como é bastante influenciado pelas águas da laguna (que induz a valores de salinidade hipohalino-mixohalinos e maiores índices de diversidade e eqüitatividade), liga-se mais fortemente à região central e nordeste do canal do Poço. Assim como as amostras 2 e 27 (canais Carapitanga e Parapuca), pode ser considerado como um ambiente de transição entre biofácies.

A Figura 9 ilustra a distribuição espacial das biofácies reconhecidas ao longo da área estudada.

\section{CONCLUSÕES}

Este estudo oferece subsídios para a compreensão do funcionamento hidroquímico e hidrodinâmico do delta do rio São Francisco, no extremo leste de Sergipe. Nas imediações de Ponta dos Mangues, águas marinhas penetram pelo inlet natural Boca do Poço e impactam fortemente o setor sudoeste do canal lagunar do Poço; a partir dali distribuem-se pelo interior do ecossistema por uma complexa rede de canais. No setor nordeste, o canal Parapuca é importante via de águas doces que, provindas do rio São Francisco, penetram a área e se somam a outras massas de água doce que aportam no sistema.

Três domínios ambientais distintos são expressos pelas biofácies Quinqueloculina spp., Ammonia tepida/Elphidium spp. e Miliammina fusca/Arenoparrela mexicana. A salinidade é fator de primeira ordem no controle da distribuição dessas biofácies, as quais ocupam, respectivamente, faixas euhalinas, hipohalino-mixohalinas e hipohalinosalobras. O cruzamento das variáveis abióticas com as bióticas, tais como dominância, diversidade, eqüitatividade e confinamento, mostra-se bastante eficiente na caracterização ambiental. 
Enquanto nas faixas hipohalino-salobras (canais Parapuca e Carapitanga) são registrados os menores valores de diversidade e eqüitatividade dos foraminíferos e os maiores valores do índice de confinamento ambiental, no domínio euhalino (área lagunar nas imediações de Ponta dos Mangues) tem-se um quadro exatamente inverso. Nas zonas hipohalinomixohalinas (região do riacho do Ostra e porção central e nordeste da laguna) registram-se valores intermediários em relação às outras duas faixas.

Ambientes dominados por rotaliinos e miliolinos correspondem àqueles mais abertos à influência marinha (biofácies Quinqueloculina spp. e Ammonia tepida/ Elphidium spp.), enquanto que nos meios hipohalinosalobros ocorrem, exclusivamente, textulariinos, alogromiinos e tecamebas arceláceas.

O padrão de distribuição dos microrganismos, o forte controle exercido pela salinidade junto às associações microbióticas e os valores de diversidade dos foraminíferos na área estudada são similares àqueles observados em outros ambientes parálicos brasileiros (e.g., Closs \& Madeira, 1967; Brönnimann et al., 1981a,b; Eichler \& Bonetti, 1995; Debenay et al. 1998; Oliveira, 1999; Eichler, 2001, Duleba \& Debenay, 2003). Há entre tais áreas, todavia, ligeiras diferenças quanto ao quadro das espécies dominantes.

Os resultados obtidos oferecem subsídios para estudos de diagnóstico e avaliação da vulnerabilidade ambiental dessa área do delta do rio São Francisco e podem ser utilizados em análises comparativas com áreas similares impactadas, ou não, por ação antrópica. Aplicam-se, ainda, em estudos de reconstrução paleoambiental de bacias sedimentares.

\section{AGRADECIMENTOS}

Os autores prestam seus agradecimentos à FAPESP, pelo financiamento da bolsa de mestrado a que se refere este projeto (processo 01/11786-8); à coordenação regional do Projeto Tamar Sergipe-Alagoas pelo apoio às atividades de campo; ao Dr. Antonio Camargo e a Carlos Sanches (LEA/ UNESP /Rio Claro) pela contribuição na obtenção de dados de salinidade em laboratório; e aos técnicos do CENPES/ PETROBRAS, CCDM/UFSCar e IFSC/USP pelo auxílio na obtenção das imagens em MEV.

\section{REFERÊNCIAS BIBLIOGRÁFICAS}

Alve, E. 1991. Benthonic foraminifera in sediment cores reflecting heavy metal pollution in Sorfjord, Western Norway. Journal of Foraminiferal Research, 21(1):1-19.

Alve, E. 1995. Benthic foraminiferal responses to estuarine pollution: a review. Journal of Foraminiferal Research, 25(3):190-203.

Alve, E. \& Murray, J.W. 1995. Benthic foraminiferal distribution and abundances changes in Skagerrak surface sediments: 1937 (Höglund) an 1992/1993 data compared. Marine Micropaleontology, 25:269-288.

Alve, E. \& Murray, J.W. 1999. Marginal marine environments of the Skagerrak and Kattegat: a baseline study of living (stained) benthic foraminiferal ecology. Palaeogeography, Palaeoclimatology, Palaeoecology, 146:171-193.

Barbosa, C.F. 1995. Foraminifera and Arcellacea ("Thecamoebia") Recentes do Estuário de Guaratuba, Paraná, Brasil. Anais da Academia Brasileira de Ciências, 67(4) :465-492.

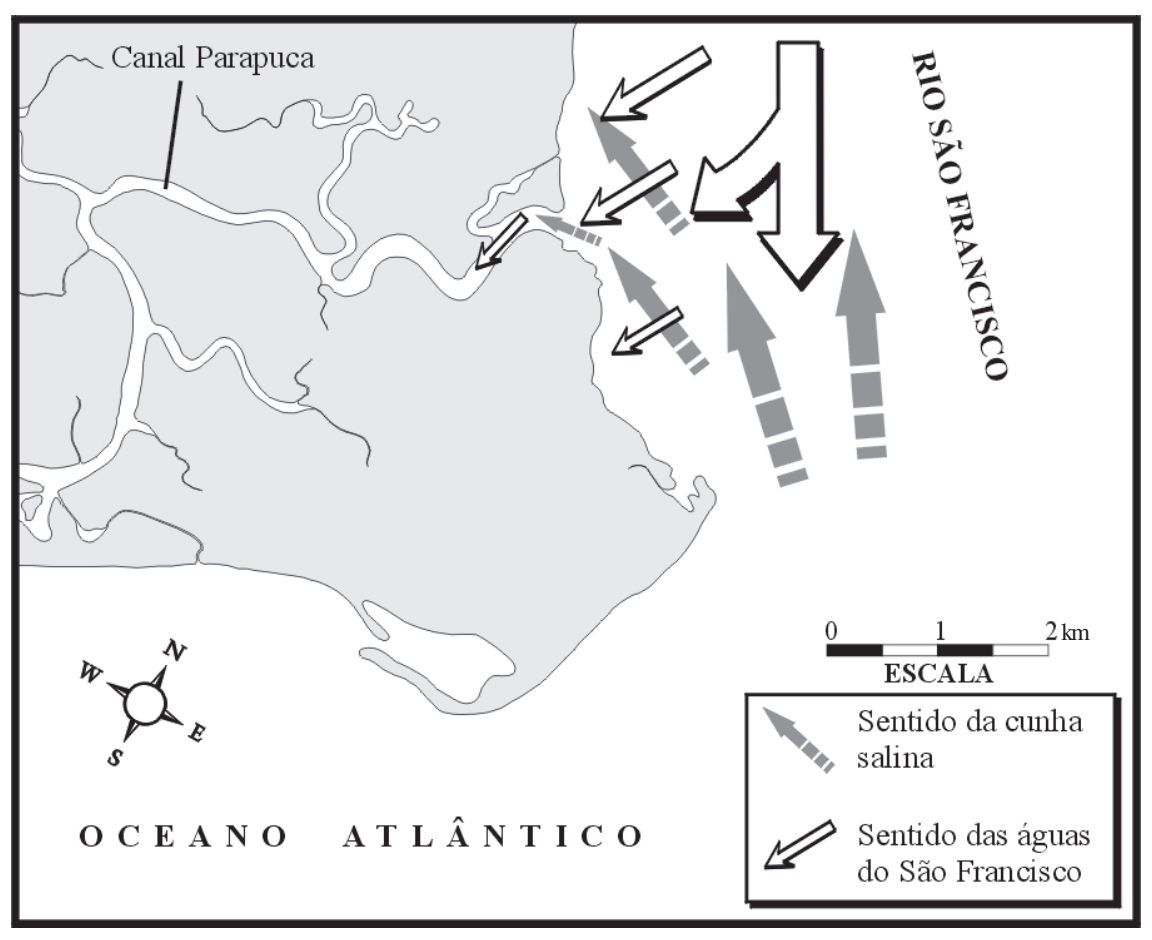

Figura 8. Modelo ilustrativo da interação entre a cunha salina e as águas do rio São Francisco.

Figure 8. Illustrative model about interaction between salt-water wedge and freshwater from São Francisco river. 


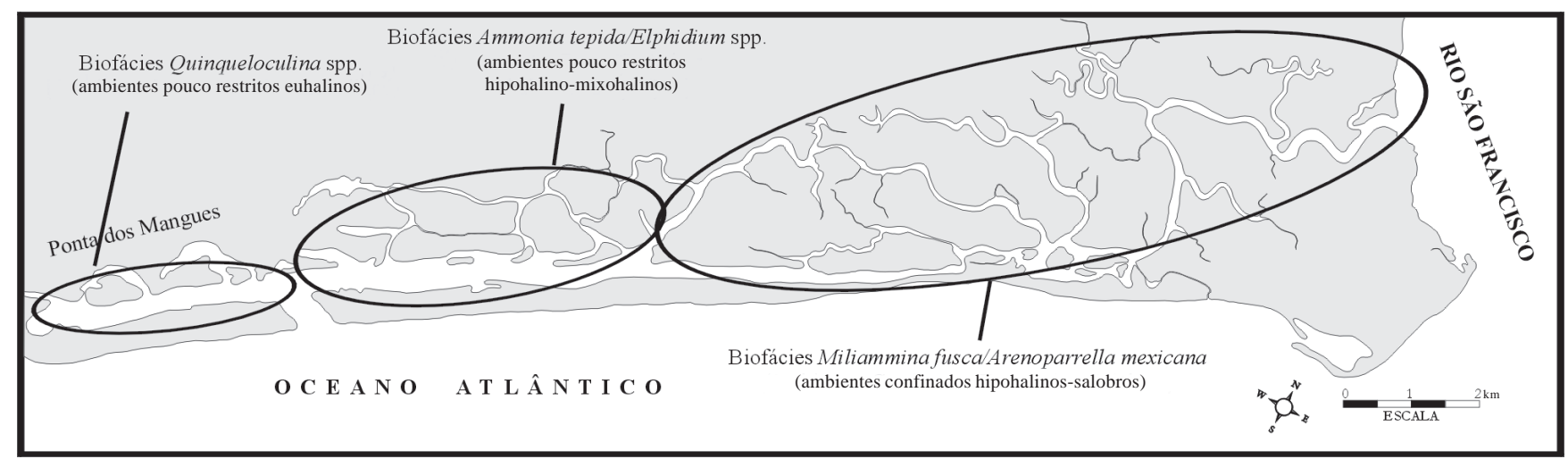

Figura 9. Divisão do setor em três biofácies, baseada na integração entre variáveis bióticas e abióticas.

Figure 9. Division of the sector in three biofacies, based on integration between biotic and abiotic variables.

Boltovskoy, E. 1965. Los Foraminiferos Recientes. Buenos Aires, Ed. Eudeba, 510 p.

Boltovskoy, E.; Giussani, G.; Watanabe, S. \& Wright, R. 1980. Atlas of benthic shelf foraminifera of the Southwest Atlantic. Holanda, Dr. W. Junk bv Publishers, The Hague, 147 p.

Bonetti, C.V.D.H. 1995. Associações de foraminíferos e tecamebas indicadoras de sub-ambientes na zona estuarina do Rio Itapitangui - Cananéia/SP. Programa de Pós-Graduação em Oceanografia Biológica, Universidade de São Paulo, Dissertação de Mestrado, 150 p.

Brönnimann, P.; Moura, J.A. \& Dias-Brito, D. 1981a. Estudos ecológicos da Baía de Sepetiba, Rio de Janeiro, Brasil: foraminíferos. In: CONGRESSO LATINO-AMERICANO DE PALEONTOLOGIA, 2, 1981. Anais, Porto Alegre, UFRGS, p.861-875.

Brönnimann, P.; Dias-Brito, D. \& Moura, J.A. 1981b. Foraminíferos da fácies mangue da planície de maré de Guaratiba, Rio de Janeiro, Brasil. In: CONGRESSO LATINO-AMERICANO DE PALEONTOLOGIA, 2, 1981. Anais, Porto Alegre, UFRGS, p.877-891.

Brönnimann, P. \& Dias-Brito, D. 1982. New lituolacea (Protista, Foraminifera) from shallow waters of the Brazilian Shelf. Journal of Foraminiferal Research, 12(1):13-23.

Brönnimann, P.; Whittaker, J.E. \& Zaninetti, L. 1992. Brackish water foraminifera from mangrove sediments of southwestern Viti Levu, Fiji Islands, Southwest Pacific. Revué de Paléobiologie, 11(1):13-65.

Closs, D. 1962. Foraminíferos e tecamebas da Lagoa dos Patos (RS). Boletim da Escola de Geologia de Porto Alegre, 11:1-130.

Closs, D. \& Madeira, M.L. 1966. Foraminifera from the Paranaguá Bay, State of Paraná, Brazil. Boletim da Universidade Federal do Paraná. Zoologia II, 10:139-162.

Closs, D. \& Madeira, M.L. 1967. Foraminíferos aglutinantes da Lagoa de Tramandaí, Rio Grande do Sul. Iheringia, 35:7-31.

Debenay, J.-P. 1990. Recent foraminiferal assemblages and their distribution relative to environmental stress in the paralic environments of west Africa (Cape Timiris to Ebrie Lagoon). Journal of Foraminiferal Research, 20(3):267-282.

Debenay, J.-P.; Eichler, B.B.; Duleba, W.; Bonetti, C. \& EichlerCoelho, P. 1998. Water stratification in coastal lagoons: its influence on foraminiferal assemblages in two Brazilian lagoons. Marine Micropaleontology, 35:67-98.
Debenay, J.-P.; Duleba, W.; Bonetti, C.; Melo-e-Souza, S.H. \& Eichler, B.B. 2001a. Pararotalia cananeiaensis n. sp., indicator of marine influence and water circulation in Brazilian coastal and paralic environments. Journal of Foraminiferal Research, 31(2):152-163.

Debenay, J.-P.; Geslin, E.; Eichler, B.B.; Duleba, W.; Sylvestre, F. \& Eichler, P. 2001b. Foraminifera assemblages in a hypersaline lagoon: the Lagoon of Araruama (RJ), Brazil. Journal of Foraminiferal Research, 31(2):133-151.

Dias-Brito, D. 1999. Plant distribution in the intertidal and supratidal zones of a mangrove-lagoon-barrier island coastal complex (São Francisco River Delta, Noertheastern Sergipe, NE Brazil). In: CONGRESSO DA ABEQUA, 7, 1999. Anais, Porto Seguro, ABRH, publicação em CD - arquivo: viiabequa_tmp009. pdf, Porto Seguro.

Dias-Brito, D.; Moura, J.A. \& Würdig, N. 1988. Relationships between ecological models based on Ostracodes and Foraminifers from Sepetiba Bay (Rio de Janeiro, Brazil). In: T. Hanay; N. Ikeya \& K. Ishizaki (eds.) Evolutionary Biology of Ostracoda, Elsevier, p. 467-484.

Dias-Brito, D. \& Rodrigues, J. 2000. Manguezal do Canal Parapuca, Delta do Rio São Francisco, Sergipe, Brasil: reflexões com base no estudo de plantas e microrganismos. In: SIMPÓSIO DE ECOSSISTEMAS BRASILEIROS, 5, 2000. Livro de Resumos, Vitória, Academia Brasileira de Ciências, p. 23-24.

Donnici, S.; Barbero, R.S.; \& Taroni, G.1997. Living benthic foraminifera in the Lagoon of Venice (Italy): population dynamics and its significance. Micropaleontology, 43(4):440-454.

Duleba, W. \& Debenay, J.-P. 2003. Hydrodynamic circulation in the estuaries of Estação Ecológica Juréia-Itatins, Brazil, inferred from foraminifera and thecamoebian assemblages. Journal of Foraminiferal Research, 33(1):62-93.

Eichler, B.B. \& Bonetti, C. 1995. Distribuição de foraminíferos e tecamebas ocorrentes no manguezal do Rio Baguaçu, Cananéia, São Paulo - Relações com parâmetros ambientais. Pesquisas, 22(1-2):35-40.

Eichler, B.B.; Debenay, J.-P.; Bonetti, C. \& Duleba, W. 1995. Répartition des foraminifères dans la zone Sud-Ouest du système laguno-estuarien d'Iguape-Cananéia (Brésil). Boletim do Instituto Oceanográfico, 43(1):1-17.

Eichler, P.P.B. 2001. Avaliação e diagnóstico do Canal de Bertioga (São Paulo, Brasil) através da utilização de foraminíferos como indicadores ambientais. Programa de Pós-Graduação em Oceanografia Biológica, Universidade de São Paulo, Tese de Doutorado, 240 p. 
Geslin, E.; Debenay, J.-P.; Duleba, W. \& Bonetti, C. 2002. Morphological abnormalities of foraminiferal tests in Brazilian environments: comparison between polluted and non-polluted areas. Marine Micropaleontology, 40(3):151168.

Hayward, B.W. \& Hollis, C. 1994. Brackish foraminifera in New Zealand: a taxonomic and ecologic review. Micropaleontology, 40(3): $185-222$.

Laidler, R.B. \& Scott, D.B. 1996. Foraminifera and Arcellacea from Porters Lake, Nova Scotia: modern distribution and paleodistribution. Canadian Journal of Earth Sciences, 33:1410-1427.

Leipnitz, I.I.; Nowatzki, C.H.; Leipnitz, B.; Aguiar, E.S.; Oliveira, R.F.G. \& Giovanoni, L. 2003. Foraminíferos do Quaternário da Lagoa do Peixe, Estado do Rio Grande do Sul, Brasil. Revista Brasileira de Paleontologia, 5:39-47.

Loeblich-Jr., A.R. \& Tappan, H. 1964. Part C, Protista 2, Sarcodina, chiefly "Thecamoebians" and Foraminiferida. In: R. C. Moore (ed.) Treatise on Invertebrate Paleontology, Lawrence, The University of Kansas Press, 900 p.

Loeblich-Jr., A.R. \& Tappan, H. 1988. Foraminiferal genera and their classification. Nova Iorque, Van Nostrand Reinhold, v. 1 e 2, 970 p. +212 p.

Magurran, A.E. 1988. Ecological diversity and its measurement. Princeton University Press, New Jersey, $179 \mathrm{p}$.

McCarthy, F.; Collins, E.; McAndrews, J.H.; Kerr, H.A.; Scott, D.B. \& Medioli, F.S.A. 1995. A comparison of postglacial Arcellacean ("Thecamoebian") and pollen succession in Atlantic Canada, illustrating the potential of Arcellaceans for paleoclimatic reconstrutions. Journal of Paleontology, 69(5):980-993.

McIntosh, R.P. 1967. An index of diversity and the relations of certain concepts of diversity. Ecology, 48:392-404.

Ogden, C.G. \& Hedley, R.H. 1980. An atlas for freshwater testate amoebae. Oxford University Press, British Museum (Natural History), $222 \mathrm{p}$.

Oliveira, D. 1999. Análise Ambiental dos canais da bacia hidrográfica do Rio Itanhaém - SP, Brasil, com base em tecamebas e foraminíferos. Programa de Pós-Graduação em Geociências e Meio Ambiente, Universidade Estadual Paulista, Dissertação de Mestrado, 252 p.

Oliveira, D. \& Dias-Brito, D. 1999. Modern thecamoebians from a coastal hydrographic basin, Southeastern Brazil. In: CONGRESSO DA ABEQUA, CUSHMAN FOUNDATION RESEARCH SYMPOSIUM, 7, 1999. Anais, Porto Seguro, ABRH, publicação em CD - arquivo: viiabequa_ccp001.pdf.

Patterson, R.T. \& Fishbein, E. 1989. Re-examination of the statistical methods used to determine the number of points counts needed for micropaleontological quantitative research. Journal of Paleontology, 63(2):245-248.

Patterson, R.T. \& Kumar, A. 2000a. Assessment of Arcellacean (Thecamoebian) assemblages, species, and strains as contaminant indicators in James Lake, Northeastern Ontario, Canada. Journal of Foraminiferal Research, 30(4): 310-320.

Patterson, R.T. \& Kumar, A. 2000b. Use of Arcellacea to gauge levels of pollution and remediation of industrially polluted lakes. In: R. E. Martin (ed.) Environmental Micropaleontology, 15: Topics in Geobiology, Kluwer Academic/Plenum Publication, p. $257-278$.
Patterson, R.T.; Dalby, A.; Kumar, A.; Henderson, L.A. \& Boudreau, R.E.A. 2002. Arcellaceans (thecamoebians) as indicators of land-use change: settlement history of the Swan Lake area, Ontario as a case of study. Journal of Paleolimnology, 28(3):297-316.

Pielou, E. C. 1969. Mathematical Ecology. Nova Iorque, John Wiley and Sons, $286 \mathrm{p}$.

Rodrigues, J.F. 1999. Distribuição de foraminíferos e tecamebas no Canal Parapuca, Delta do Rio São Francisco, Sergipe. Graduação em Ecologia, Universidade Estadual Paulista, Trabalho de Conclusão, 59 p.

Santos, M.M. 1997. Ponta dos Mangues: relação sociedadenatureza. Programa de Pós-Graduação em Desenvolvimento e Meio Ambiente, Universidade Federal de Sergipe, Dissertação de Mestrado, $112 \mathrm{p}$.

Schafer, C.T.; Winters, G.V.; Scott, D.B.; Pocklington, P.; Cole, F.E. \& Honig, C. 1995. Survey of living foraminifera and polychaete populations at some canadiam aquaculture sites: potential for impact mapping and monitoring. Journal of Foraminiferal Research, 25(3):243-259.

Scott, D.B. \& Medioli, F.S. 1980. Quantitative studies of marsh foraminiferal distributions in Nova Scotia: implications for sea level studies. Cushman Foundation for Foraminiferal Research (Special Publication), 17:1-58.

Scott, D.B.; Schnack, E.J.; Ferrero, L.; Espinosa, M. \& Barbosa, C.F. 1990. Recent marsh foraminifera from the East Coast of South America: comparison to the Northern Hemisphere. In: C. Hemleben (ed.) Paleoecology, Biostratigraphy, Paleoceanography and Taxonomy of Agglutinated Foraminifera, Holanda, Kluwer Academic Publishers, p. 717-737.

Scott, D.B.; Medioli, F.S. \& Schafer, C.T. 2001. Monitoring of coastal environments using Foraminifera and Thecamoebian indicators. Cambridge University Press.

Semensatto-Jr, D.L. 2001. Foraminíferos e tecamebas do Canal do Poço, Sergipe: distribuição e condicionantes. Graduação em Ecologia, Universidade Estadual Paulista, Trabalho de Conclusão, 45 p.

Semensatto-Jr., D.L. 2003. Aplicação de índices de diversidade em estudos envolvendo associações entre foraminíferos e tecamebas recentes: uma breve discussão. In: CONGRESSO DA ASSOCIAÇÃO BRASILEIRA DE ESTUDOS DO QUATERNÁRIO, 9, 2003. Anais (publicação em CD-ROM), Recife.

Sharifi, A.R.; Croudace, I.W. \& Austin, R.L. 1991. Benthic foraminiferids as pollution indicators in Southampton Water, southern England, U. K. Journal of Micropaleontology, 10(1):109-113.

Stevenson, M.R.; Dias-Brito, D.; Stech, J.L. \& Kampel, M. 1998. How do cold biota arrive in a tropical bay near Rio de Janeiro, Brazil? Continental Shelf Research, 18:1595-1612.

Todd, R. \& Low, D. 1981. Marine flora and fauna of the Northeastern United States. Protozoa: Sarcodina: Benthic foraminifera. Rockville (MD): NOAA/MNFS, 50, circ. 439.

Wetmore, K.L. 1995. Foraminifera: life and ecology. University of California, Museum of Paleontology, Berkeley, Estados Unidos, Disponível em: www.ucmp.berkeley.edu/foram/foramlh.html. Acesso em: 08/01/2001.

Zaninetti, L.; Brönnimann, P.; Beurlen, G. \& Moura, J.A. 1977. La mangrove de Guaratiba et la Baie de Sepetiba, état de Rio de Janeiro, Brésil: foraminifères at écologie. Archive dês Sciences de Gèneve, 30(2):161-178. 
Zaninetti, L.; Brönnimann, P.; Dias-Brito, D.; Arai, M.; Casaletti, P.; Koutsoukos, E. \& Silveira, S. 1979. Distribuition écologique des Foraminifères dans la mangrove dáAcupe, Etat de Bahia, Brésil. Notes du Laboratoire de Paléontologie de L'université de Genève, 4(1):1-17.
Zucon, M.H. \& Loyola e Silva, J. 1992. Distribuição de foraminíferos e tecamebas no estuário do Rio Piauí, Sergipe. Nerítica, 7(1/2):57-69.

Received October, 2003; accepted January, 2004. 